\title{
System reconfiguration of large-scale control systems using back-up actuators
}

\author{
Carlos Trapiello ${ }^{1,2}$, Vicenç Puig ${ }^{1,2}$ and Gabriela Cembrano ${ }^{1,2}$
}

\begin{abstract}
The intrinsic redundancy of large-scale control systems allows to differentiate between the nominal system configuration, where the dynamics of the plant are controlled through a specific subset of actuators, and the possible configurations that arise from the combination of all the existing actuators (nominal plus back-up ones). After the occurrence of an unexpected event (component fault, isolation of a specific area due to security concerns, etc), some of the back-up system actuators can be used in order to guarantee that the system performance will remain within some admissible margins. Thus, given a scenario that causes a non-admissible system operation with the nominal configuration, the possibility to use back-up actuators in order to improve the system performance expands the traditional fault tolerant control scenario defining a new problem. Accordingly, this work is devoted to the introduction of the problem which is solved by formulating the actuator selection as a mixed-integer programming optimization. A water distribution network is used with the aim to illustrate the new problem implications as well as to demonstrate the validity of the proposed solution.
\end{abstract}

\section{INTRODUCTION}

Large-scale control systems are exposed to incidents that may disrupt the system nominal operation such as: component fault, planned interruptions due to periodic maintenance, isolation of a part of the network to stop the propagation of a threat, etc. An adequate management of such situations becomes fundamental in order to protect the system integrity and accelerate the restoration to its nominal operation. The fact that large-scale systems tend to present certain physical redundancy can be exploited in order to manage performance-compromising scenarios, and, by means of a proper system reconfiguration, inadmissible effects on the system performance may be prevented for a sufficiently long period of time.

From an automatic control perspective, the management of such situations is placed within the fault tolerant control (FTC) problem. Usually, FTC is divided into passive FTC schemes, which work with a fixed controller that provide robust properties under certain faults, and active FTC schemes, which restructure the control loop and adapt the controller to the faulty plant [1]. Traditionally, active FTC advances have focused in achieving fault accommodation by exploiting the analytical redundancy of the system variables for a fixed

This work has been partially funded by AGAUR ACCIO RIS3CAT UTILITIES 4.0 - P7 SECUTIL and by SMART Project (ref. num. EFA153/16 Interreg Cooperation Program POCTEFA 2014-2020).

1 Research Center for Supervision, Safety and Automatic Control (CS2AC), Universitat Politècnica de Catalunya (UPC), Spain carlos.trapiello@upc.edu.

2 Institut de Robòtica i Informàtica Industrial, CSIC-UPC, Llorens i Artigas 4-6, 08028 Barcelona, Spain. sensors-actuators structure, while the term physical redundancy was relegated to consider duplication of hardware components, and hence, reducing the FTC problem into the switching of the faulty component by a healthy one [2].

Nevertheless, little attention has been paid to the fact that, apart from nominal set of actuators, large-scale systems (specially network systems) may exhibit alternative connections and actuators that can be used under some circumstances, and where the capability to modify the input structure with the addition of new control variables extends the previous definition of active FTC. This redundant structure appears in water distribution networks (WDNs), where the control of the water tanks levels and demand flow satisfaction is achieved by means of a nominal set of valves and pumps. However, large WDNs usually include a set of alternative valves (normally closed), pumps (normally off) and secondary pipes that could be used in an emergency. The use of a subset of such alternative actuators, may yield a reconfigured system that presents an admissible performance in a situation where the nominal system performance becomes inadmissible.

The system reconfiguration problem is a well-known problem in power grids. The so-called power distribution network reconfiguration (PDNR) problem, studies the system switches disposition in order to reduce power loss, increase system security and/or enhance power quality [3]. However, the binary structure of the switches has led to specific algorithms for PDNR [4], which are hard to extrapolate to more general cases. From a strictly automatic control perspective, there are few references that address this problem. Among the most remarkable ones, there is Chapter 4 of [5] where the reconfiguration problem is addressed within a mixed logical dynamical (MLD) framework: I) together with the control using a predictive control scheme; II) with a two decision level procedure, a first level where the set of new inputs used for control are selected, and a second level where a receding control strategy including the new inputs is applied.

The presented problem can also be treated as a control allocation problem (CAP) [6], [7], which should be extended with the selection of the appropriate actuators under certain optimality criteria. Besides, the varying input structure of the system reconfiguration problem, resembles some of the challenges faced by plug \& play control schemes [8]. Referring back to WDNs, works like [9] provide a response methodology for reducing the impact of failure situations, where the selection among a possible set of interventions relies on genetic algorithms.

According to the aforementioned background information, this paper is devoted to the introduction of the system 
reconfiguration problem in the sense of selecting the appropriate hardware components after the occurrence of an incident and subject to the fulfilment of certain performance conditions. The extraordinary circumstances that trigger the reconfiguration problem define a clear hierarchy in the control objectives, prioritizing the required reconfiguration effort or safety related metrics over other objectives like, for example, economic objectives. Therefore, the actuator selection is addressed using a lexicographic approach, where the combinatorial nature of the problems lead to mixedinteger programming (MIP) optimizations which are known to be NP-complete, with a worst-case optimization time that scales exponentially with problem size. Several heuristics can be applied in order to reduce the number of explored nodes in the MIP problem. The aim of this formulation is to provide a support tool that helps system operators to efficiently deal with this type of situations. Consequently, in the present paper, the actuators selection is based on an open loop optimization for systems modelled as discrete linear difference-algebraic equations. A small portion of a WDN is used in order to exemplify the problem.

The remainder of the paper is organized as follows: Section II formulates de problem statement. The actuators selection method is presented in Section III. Section IV, introduces the case study considered, and the obtained results are analysed in Section V. Finally, the main conclusions are drawn in Section VI.

\section{PROBLEM STATEMENT}

In order to illustrate the problem, let us consider the control-oriented model of a WDN which may be described by means of a set of linear (or linearised) discrete differencealgebraic equations (DAE) [10], for all time instant $k \in \mathbb{N}$

$$
\begin{aligned}
x_{k+1} & =A x_{k}+B u_{k}+B_{d} d_{k} \\
0 & =E u_{k}+E_{d} d_{k}
\end{aligned}
$$

where the difference equation (1a) describes the dynamics of the plant, and the algebraic equations in (1b) describe the static relations in the network. Vector $x_{k} \in \mathbb{R}^{n_{x}}$ denotes the state variables, $u_{k} \in \mathbb{R}^{n_{u}}$ the control actions and $d_{k} \in \mathbb{R}^{n_{d}}$ represents the demanded flow modelled as additive measured disturbances. Moreover, $A, B, B_{d}, E, E_{d}$, are time-invariant matrices of suitable dimensions dictated by the network topology. Let us denote as $\mathscr{S}_{n}\left(A, B, B_{d}, E, E_{d}\right)$, or simply $\mathscr{S}_{n}$, the nominal system structure (1a)-(1b).

Assumption 1: The states $x_{k}$ and demands $d_{k}$ are observable at time instant $k$, and the pair $(A, B)$ is stabilisable.

Furthermore, the system is subject to state and control constraints $\forall k$

$$
u_{k} \in \mathscr{U}, \quad x_{k} \in \mathscr{X}
$$

where $\mathscr{U} \subset \mathbb{R}^{n_{u}}$ is compact and $\mathscr{X} \subset \mathbb{R}^{n_{x}}$ is closed.

Throughout this work it is also assumed that a diagnosis block, or a human operator in case for example of a maintenance routine, feeds the tolerant control scheme with a reasonable estimation of the incidence (or event) affecting the system. In this regard, let us consider the set
$\mathscr{E}=\left\{f_{i}, \ldots, m_{i}, \ldots, a_{i}, ..\right\}$ that encompasses all possible events that may affect the system, including: possible component faults $f_{i}$, maintenance routines $m_{i}$, physical or cyber attacks $a_{i}$, etc. The effect of such events is multiplicatively modelled affecting the system matrices.

Considering the presence of the diagnosis block, the following temporal sequence is defined: an event occurs at time $k_{0}$, the event is diagnosed at $k_{d} \geq k_{0}$ and the appropriate interventions are immediately carried out. Depending on the nature of the event $e \in \mathscr{E}$, a time horizon $N$ is considered such that the normal restoration of the service is expected before the sample $k_{f}=k_{d}+N$.

Assumption 2: Unexpected events are immediately diagnosed, i.e. $k_{d}=k_{0}$.

Hence, after the occurrence of an event at $k_{0}$, for $k \geq k_{0}$ the system will evolve according to

$$
\begin{aligned}
x_{k+1}^{e} & =A(e) x_{k}^{e}+B(e) u_{k}^{e}+B_{d}(e) d_{k} \\
0 & =E(e) u_{k}^{e}+E_{d}(e) d_{k}
\end{aligned}
$$

starting at $x_{k_{0}}$. Let us denote as $\mathscr{S}_{e}$ the system structure (3a)(3b) after the occurrence of $e \in \mathscr{E}$.

Note that the shut down of the $i^{\text {th }}$ actuator implies that the corresponding column of the input matrices $B(e)$ and $E(e)$ are set to zero.

The decision whether or not use some of the existing alternative actuators is based on the expected $\mathscr{S}_{e}$ performance during the corresponding time horizon $N$. Below, the admissibility performance conditions and the considered actuators classification are presented.

\section{A. Admissibility condition}

Let us consider a reference system evolving according to

$$
x_{k+1}^{r}=A x_{k}^{r}+B u_{k}^{r}+B_{d} \hat{d}_{k}
$$

where $\hat{d}_{k}$ is the flow demand prediction at time $k$ inferred from the periodic patterns of the network. Based on the reference dynamics (4), the user may define several quantitative factors in order to assess the system operation. In the present work, the system performance will be evaluated according to

$$
\begin{aligned}
& J_{N}\left(\mathscr{S}, x_{k_{0}} \tilde{x}^{r}, \tilde{d}, \tilde{u}\right)= \\
& =\sum_{j=1}^{N} \|\left(x_{j}^{r}-\hat{x}\left(k_{0}+j \mid k_{0}\right)\left\|_{Q}^{2}+\sum_{j=0}^{N-2}\right\| u(j+1)-u(j) \|_{R}^{2}\right.
\end{aligned}
$$

where the considered quadratic cost is a function of the system evolution $\mathscr{S}$, the initial state $x_{k_{0}}$, the sequences of reference state $\tilde{x}^{r}=\left(x_{k_{0}}^{r}, \ldots, x_{k_{f}}^{r}\right)$, predicted demands $\tilde{d}=\left(\hat{d}_{k_{0}}, \ldots, \hat{d}_{k_{f}-1}\right)$ and the computed control actions $\tilde{u}=$ $(u(0), \ldots, u(N-1))$. The vector $\hat{x}(k+j \mid k)$ denotes the prediction of the state at time $k$ and $j$ samples into the future. The weighting matrices $Q$ and $R$ are positive semi-definite with appropriate dimensions.

After the occurrence of an event $e \in \mathscr{E}$ at $k_{0}$, the expected performance $\alpha_{N}\left(\mathscr{S}_{e}, x_{k_{0}}\right)$ during the horizon $N$ for system $\mathscr{S}_{e}$ is computed as 


$$
\begin{array}{lll}
\alpha_{N}\left(\mathscr{S}_{e}, x_{k_{0}}\right)= & \min _{\tilde{u}} J_{N}\left(\mathscr{S}_{e}, x_{k_{0}}, \tilde{x}^{r}, \tilde{d}\right) \\
\text { s.t. } & \hat{x}\left(k_{0}+j+1 \mid k_{0}\right)=g\left(u(j), \hat{x}\left(k_{0}+j \mid k_{0}\right), \mathscr{S}_{e}, \hat{d}\right) \\
& \hat{x}\left(k_{0} \mid k_{0}\right)=x_{k_{0}} & \\
& \hat{x}\left(k_{0}+j \mid k_{0}\right) \in \mathscr{X} & \forall j \in\{0, \ldots, N\} \\
& u(j) \in \mathscr{U} & \forall j \in\{0, \ldots, N-1\}
\end{array}
$$

with $\hat{x}\left(k_{0}+j+1 \mid k_{0}\right)=g\left(u(j), \hat{x}\left(k_{0}+j \mid k_{0}\right), \mathscr{S}_{e}, \hat{d}\right)$ representing the system evolution according to (3a)-(3b).

Consequently, the condition that validates the admissibility of a faulty system is formulated as

$$
\alpha_{N}\left(\mathscr{S}_{e}, x_{k_{0}}\right) \leq \sigma_{N}\left(x_{k_{0}}\right)
$$

where $\sigma_{N}\left(x_{k_{0}}\right) \in \mathbb{R}$ is a threshold value selected by the user.

A natural way to set the admissible performance threshold is by referring it to the expected nominal performance: $\sigma_{N}\left(x_{k_{0}}\right)=\beta \cdot \alpha_{N}\left(\mathscr{S}_{n}, x_{k_{0}}\right)$, with $\beta \in \mathbb{R}$ such that $\beta \geq 1$ (it is to be expected that the system performance in an abnormal situation will be worse than the nominal one).

If condition (7) is not satisfied, a quest must be performed searching for possible system configurations that fulfil it.

\section{B. Actuators classification}

By denoting as $\mathscr{A}_{T}$ the set of all actuators (with cardinality $\left|\mathscr{A}_{T}\right|=n_{T}$ ), then, the following classification can be performed:

- $\mathscr{A}_{N} \subseteq \mathscr{A}_{T}$ is the subset of all the actuators used in the nominal operation. Note that the performance of this control elements is subject to the occurrence of an event $\left(\left|\mathscr{A}_{N}\right|=n_{N}\right)$.

- $\mathscr{A}_{E} \subseteq \mathscr{A}_{N}$ is the subset of nominal actuators that rest after the occurrence of an event $e \in \mathscr{E}\left(\left|\mathscr{A}_{E}\right|=n_{E}\right)$.

- $\mathscr{A}_{R}=\mathscr{A}_{T} \backslash \mathscr{A}_{N}$ is the subset of all other alternative actuators that can be additionally used for system reconfiguration $\left(\left|\mathscr{A}_{R}\right|=n_{R}\right)$.

- $\mathscr{A}_{C} \subseteq \mathscr{A}_{R}$ encompasses the subset of alternative actuators that are considered in the optimization stage. The selection of $\mathscr{A}_{C}$ depends on the event $e \in \mathscr{E}\left(\left|\mathscr{A}_{C}\right|=n_{C}\right)$.

- $\mathscr{A}_{A} \subseteq \mathscr{A}_{C}$ is the selected subset of alternative actuators that are temporarily used during the time horizon $N$ in order to guarantee a certain performance level $\left(\left|\mathscr{A}_{A}\right|=\right.$ $\left.n_{A}\right)$.

Note that, according to the previous definitions, the set of candidate actuators $\mathscr{A}_{C}$ is only made up of alternative actuators. Thus, in this case, the set of already used actuators $\mathscr{A}_{E}$ is maintained and not included in the selection process.

Two remarks must be made: 1) an appropriate selection of the candidate actuators $\mathscr{A}_{C}$ (among all the possible alternatives $\mathscr{A}_{R}$ ) may accelerate the convergence time of the optimization stage. Knowledge of the network dynamics can be used in this step to get rid of non consistent combination of actuators; 2) not all the actuators in $\mathscr{A}_{R}$ imply the same reconfiguration effort, i.e. some of them may be easily turned on (like automated valves), while others should only be employed under critical circumstances. This might be reflected in the weights associated to each actuator.

\section{ACTUATOR SELECTION}

\section{A. Control objectives during the reconfiguration stage}

After the occurrence of an event, a hierarchical list of control objectives must be established. Let us consider an example of $p$ different objectives listed in decreasing priority order

- Objective 1: minimize the number of alternative actuators used in the reconfiguration $\left(f_{1}\right)$.

- Objective 2: minimize the performance degradation within the admissibility margins $\left(f_{2}\right)$.

- ...

- Objective $p$ : the less important objective $\left(f_{p}\right)$

with $f_{i}$ being a scalar-valued objective function for all $i \in$ $\{1, \ldots, p\}$.

\section{B. Actuator selection optimization}

Let us denote as $v_{k} \in \mathbb{R}^{n_{C}}$ the values of control variables associated to the elements in the $\mathscr{A}_{C}$ set. These control variables are subject to the constraint

$$
v_{k} \in \mathscr{V}
$$

where $\mathscr{V} \subset \mathbb{R}^{n_{C}}$ is a compact set.

In order to select the elements of $\mathscr{A}_{A}$ that constitute the final set of actuators $\mathscr{A}_{F}=\mathscr{A}_{E} \cup \mathscr{A}_{A}$, an auxiliary vector $z_{k} \in$ $\mathbb{R}^{n_{C}}$ is used, such that

$$
z_{k}=\operatorname{diag}(\delta) \cdot v_{k}
$$

where $\operatorname{diag}(\cdot)$ is a square matrix with elements in the diagonal, and $\delta=\left[\delta_{1}, \ldots, \delta_{n_{C}}\right]^{T} \in\{0,1\}^{n_{C}}$ is a vector of Boolean variables that sets the use of the corresponding actuators for all $k \in\left[k_{0}, k_{f}\right]$

$$
\begin{aligned}
& {\left[\delta_{i}=0\right] \rightarrow\left[z_{k}^{i}=0\right] \quad \text { the } i^{t h} \text { actuator is not used }} \\
& {\left[\delta_{i}=1\right] \rightarrow\left[z_{k}^{i}=v_{k}^{i}\right] \quad \text { the value of the } i^{t h} \text { actuator is } v_{k}^{i}}
\end{aligned}
$$

The previous logic statements can be transformed into equivalent linear integer programs [11] as follows

$$
\begin{aligned}
\operatorname{diag}(\boldsymbol{\delta}) \underline{v} & \leq z_{k} \leq \operatorname{diag}(\boldsymbol{\delta}) \bar{v} \\
v_{k}-\operatorname{diag}(1-\delta) \bar{v} & \leq z_{k} \leq v_{k}-\operatorname{diag}(1-\delta) \underline{v}
\end{aligned}
$$

where $\bar{v}, \underline{v} \in \mathbb{R}^{n_{C}}$ represent the upper and lower bounds for the variable $v$, which can be obtained by means of the interval hull of the $\mathscr{V}$ set .

Note that the binary vector $\delta$ provides direct information regarding which actuators in $\mathscr{A}_{C}$ should be turned on. Also note that, with the previous formulation, this vector maintains the same values throughout the time horizon $N$.

With the consideration of the possible new actuators, the system will evolve according to

$$
\begin{aligned}
x_{k+1}^{a} & =A(e) x_{k}^{a}+\left[B(e) \quad B_{A_{C}}\right]\left[\begin{array}{l}
u_{k}^{a} \\
z_{k}
\end{array}\right]+B_{d}(e) d_{k} \\
0 & =\left[\begin{array}{ll}
E(e) & E_{A_{C}}
\end{array}\right]\left[\begin{array}{l}
u_{k}^{a} \\
z_{k}
\end{array}\right]+E_{d}(e) d_{k}
\end{aligned}
$$

where $B_{A_{C}}, E_{A_{C}}$ are the input matrices associated to the elements in $\mathscr{A}_{C}$. Let us denote system (12a)-(12b) as $\mathscr{S}_{a}$. 
Therefore, the actuator selection subject to the fulfilment of the admissibility conditions expressed in Section II-A, is formulated as a multi-objective optimization problem $\mathscr{P}$ of the form

$$
\begin{array}{ll}
\mathscr{P}: \min _{\delta, \tilde{u}, \tilde{v}} & f=\left[f_{1}, f_{2}, \ldots, f_{p}\right]^{T} \\
\text { s.t. } & J_{N}\left(\mathscr{S}_{a}, x_{k_{0}}, \tilde{x}^{r}, \tilde{d}\right) \leq \sigma_{N}\left(x_{k_{0}}\right) \\
& \hat{x}\left(k_{0}+j+1 \mid k_{0}\right)=g\left(u(j), z(j), \hat{x}\left(k_{0}+j \mid k_{0}\right), \mathscr{S}_{a}, \hat{d}\right) \\
& \hat{x}\left(k_{0} \mid k_{0}\right)=x_{k_{0}} \\
& v(j)-\operatorname{diag}(1-\delta) \bar{v} \leq z(j) \leq v(j)-\operatorname{diag}(1-\delta) \underline{v} \\
& \operatorname{diag}(\delta) \underline{v} \leq z(j) \leq \operatorname{diag}(\delta) \bar{v} \\
& x(j) \in \mathscr{X} \quad \forall j \in\{0, \ldots, N\} \\
& u(j) \in \mathscr{U}, v(j) \in \mathscr{V} \quad \forall j \in\{0, \ldots, N-1\}
\end{array}
$$

There are several approaches for solving multi-objective optimizations, being the most popular to form a scalar cost function composed of a linear weighted sum of the individual cost functions $f_{i}$. Nevertheless, the exceptional nature of the events that trigger the reconfiguration problem define a clear hierarchy between the different objectives, motivating thus the use of lexicographic approach in order to tackle the optimization problem $\mathscr{P}$.

\section{Lexicographic approach}

In order to present the lexicographic approach let us expresses the multi-objective problem $\mathscr{P}$ in (13) as

$$
\mathscr{P}: \min _{\theta \in \Theta} f(\theta)
$$

where the vector $\theta$ encompasses the different decision variables and $\Theta \subset \mathbb{R}^{d}$ is its admissible set. The vectorvalued objective function $f: \Theta \rightarrow \mathbb{R}^{p}$, is such that $f(\theta):=$ $\left[f_{1}(\theta), \ldots, f_{p}(\theta)\right]^{T}$ being $f_{i}: \Theta \rightarrow \mathbb{R}$ the scalar-valued objective functions defined in Section III-A.

According to [12], a given $\theta^{*} \in \Theta$ is a lexicographic minimizer and $f\left(\theta^{*}\right)$ is the lexicographic minimum of $\mathscr{P}$, if and only if there does not exist a $\theta \in \Theta$ and an $i^{*}$ satisfying $f_{i^{*}}(\theta)<f_{i^{*}}\left(\theta^{*}\right)$ and $f_{i}(\theta)=f_{i}\left(\theta^{*}\right), i=1, \ldots, i^{*}-1$.

An interpretation of the above definition is that a solution is a lexicographic minimum if and only if an objective $f_{i}$ can be reduced only at the expense of increasing at least one of the higher-prioritized objectives $\left\{f_{1}, \ldots, f_{i-1}\right\}$. Note that lexicographic solution is a special type of Pareto-optimal solution that takes into account the order of the objectives. For a problem $\mathscr{P}$ a lexicographic minimizer exists and the lexicographic minimum is unique [12].

Among the different approaches to find the lexicographic minimum, the present work uses the sequential solution method summarized in Algorithm 1 [13].

Note from step 5 of Algorithm 1 that the lexicographic minimizer is not guaranteed to be unique. A sufficient condition for guaranteeing the uniqueness of the lexicographic minimizer is that at least one of the cost functions $f_{i}$ is strictly convex and attains its minima inside $\Theta \neq \emptyset$. For this particular case, the minimizer is obtained as

$$
\boldsymbol{\theta}^{*}=\underset{\boldsymbol{\theta} \in \Theta}{\arg \min }\left\{f_{i}(\boldsymbol{\theta}) \mid f_{j}(\boldsymbol{\theta}) \leq f_{j}^{*}, j=1, \ldots, i-1\right\}
$$

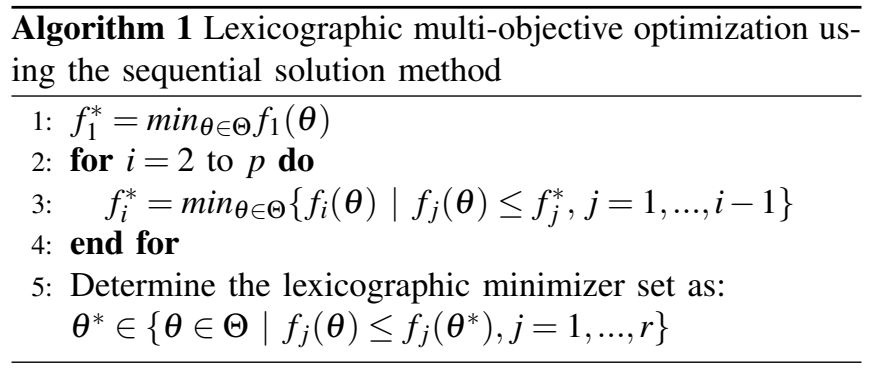

and there will be no need to continue Algorithm 1 for the remaining steps $\left\{f_{i+1}^{*}, \ldots, f_{p}^{*}\right\}$.

\section{Specific minimum lexicographic}

Let us consider the specific case that only the two first control objectives presented in Section III-A are considered. Then, this control objectives can be formulated as

$$
\begin{aligned}
& f_{1}=\|\delta\|_{m}^{1} \\
& f_{2}=J_{N}\left(\mathscr{S}, x_{k_{0}} \tilde{x}^{r}, \tilde{d}, \tilde{u}\right)
\end{aligned}
$$

where $m \in \mathbb{R}^{n_{C}}$ is a vector that weights the reconfiguration effort associated to each candidate actuator.

The binary-valued vector $\delta$, in addition with the existence of a threshold value in the objective function $f_{2}$, allows finding a lexicographic minimum by defining a weighted scalar objective function $f^{s}$ of the form [14]

$$
\mathscr{P}: \min _{\theta \in \Theta} f^{s}=\gamma \cdot f_{1}+f_{2}
$$

for $\gamma>\sigma_{N}\left(x_{k_{0}}\right)$ and the components $m_{i} \geq 1, \forall i \in\left\{1, \ldots, n_{c}\right\}$. That is, minimizing the number of required alternative actuators (given by $\delta$ ) always result in a greater reduction in cost than the minimization of the $f_{2}$ objective.

It must be noted that, by solving the optimization problem formulated in (17), the information retrieved from the obtained minimizer $\theta^{*}$ is: the configuration with minimum number of alternative actuators that guarantees the fulfilment of the admissibility condition, and for the case of more than one minimum configuration satisfying it, then, the configuration that achieves the best performance degradation within the established margins.

Despite the efficiency of modern MIP solvers, the combinatorial nature of the problem is subject to a worstcase exploration of $2^{n_{C}}$ nodes in the search tree. In some applications, there may exist a limit in the maximum number $s\left(s<n_{C}\right)$ of interventions that can be performed, e.g. manual operations with limited staff. Thus, these constraints should be added to the optimization as

$$
\sum_{i=1}^{n_{C}} \delta_{i} \leq s
$$

with the aim of limiting the extent of the search tree to $\sum_{k=0}^{s} \frac{n_{C} !}{k !\left(n_{C}-k\right) !}<2^{n_{C}}$ nodes, reducing thus, the worst execution time of each optimization. 


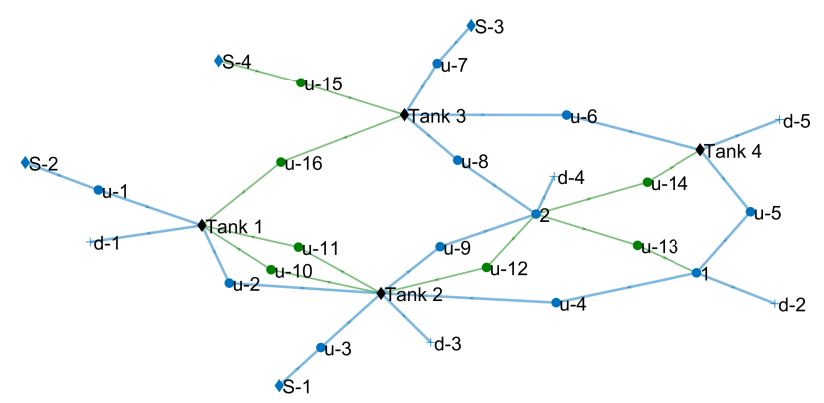

Fig. 1. Overall system representation

\section{CASE STUDY DESCRIPTION}

The considered case study is a simplified network based on a portion of the Barcelona WDN. The system under study consist of: $n_{x}=4$ tanks, $n_{d}=5$ sinks, $n_{q}=2$ nodes, $n_{s}=4$ sources and $n_{T}=16$ actuators. Thus, the set $\mathscr{A}_{T}=\{1, \ldots, 16\}$ conforms the total set of the enumerated actuators.

For academic purposes, let us assume that the first $n_{N}=9$ actuators are the ones used in nominal operation, i.e. $\mathscr{A}_{N}=\{1, \ldots, 9\}$. On the other hand, the rest of the existing actuators are considered as alternative $\mathscr{A}_{R}=$ $\{10,11,12,13,14,15,16\}$, that is, without any effect in the system nominal operation (pumps off and valves closed). Fig. 1 depicts a schematic plot of the network structure, where the nominal actuators (and their associated pipes) are plotted in blue, the alternative elements are shown in green and the system tanks are depicted in black.

From the total of $n_{T}=16$ actuators, the elements $\{1,3,7,11,15\}$ are pumps, while the rest are valves capable to regulate the flow passing through the pipe. The use of the pumps has an associated electrical cost that will be used in a planner stage, but not as a measurement of performance.

The expected demands patterns follows a cycle pattern of $24 h$ and the considered sampling time $T_{s}$ is $1 h$.

The tank volumes (system states $x \in \mathbb{R}^{4}$ ), the flow through the nominal actuators (nominal control actions $u \in \mathbb{R}^{9}$ ) and the flow passing through the alternative actuators (alternative control actions $v \in \mathbb{R}^{7}$ ), are subject to physical constraints

$$
\underline{x} \leq x \leq \bar{x} \quad \underline{u} \leq u \leq \bar{u} \quad \underline{v} \leq v \leq \bar{v}
$$

with

$$
\begin{aligned}
& \underline{x}=[198,480,0,800]^{T}\left(\left[m^{3}\right]\right) \\
& \bar{x}=[3870,3250,14450,3100]^{T}\left(\left[m^{3}\right]\right) \\
& \underline{u}=[0,0,0,0,0,0,0,0,0]^{T}\left(\left[m^{3} / s\right]\right) \\
& \bar{u}=[0.03,0.03,0.4,0.22,0.75,1.8,5.34,0.8,0.07]^{T}\left(\left[m^{3} / s\right]\right) \\
& \underline{v}=[0,0,0,0,0,0,0]^{T}\left(\left[m^{3} / s\right]\right) \\
& \bar{v}=[0.12,0.05,0.011,0.5,0.159,2.50,2.90]^{T}\left(\left[m^{3} / s\right]\right)
\end{aligned}
$$

Note that all the considered alternative actuators present certain actuation range. The presence of on-off valves could be modelled proceeding as detailed in Section III-B.

\section{A. Reference model}

As stated in Section II, the system performance is compared with respect to a reference model. The design of the reference trajectories is done following an economic MPC planner, where the cyclic behaviour of water consumption and electricity cost are used in order solve an open-loop optimization, minimizing the associate cost of the plant operation [15].

The obtained trajectory exhibit a $24 h$ periodic pattern that takes advantage of the lower electricity prices to fill the deposits during the night.

TABLE I

PARAMETER VALUES FOR THE DIFFERENT SCENARIOS

\begin{tabular}{cccccc}
\hline Scenario & $\boldsymbol{k}_{\boldsymbol{0}}$ & $\mathbf{N}$ & $\boldsymbol{\beta}$ & $\mathscr{A}_{\boldsymbol{E}}$ & $\mathscr{A}_{\boldsymbol{C}}$ \\
\hline 1 & $10 \mathrm{~h}$ & $24 \mathrm{~h}$ & 1.5 & $\mathscr{A}_{N}$ & $\mathscr{A}_{R}$ \\
2 & $4 \mathrm{~h}$ & $24 \mathrm{~h}$ & 1.5 & $\mathscr{A}_{N} \backslash\{4,9\}$ & $\mathscr{A}_{R}$ \\
3 & $4 \mathrm{~h}$ & $24 \mathrm{~h}$ & 1.5 & $\mathscr{A}_{N} \backslash\{4,9\}$ & $\mathscr{A}_{R} \backslash\{12\}$ \\
4 & $2 \mathrm{~h}$ & $6 \mathrm{~h}$ & 1.5 & $\mathscr{A}_{N}$ & $\mathscr{A}_{R}$ \\
\hline
\end{tabular}

\section{B. Scenarios}

Different scenarios were tested in order to validate the proposal. The considered parameter values for each scenario are listed in Table I.

- Scenario 1: (Component fault) The first scenario considers a multiplicative fault $f=0.2$ in the $6^{\text {th }}$ actuator of the system, i.e. the sixth columns of the input matrices are multiplied by the factor $f$.

- Scenarios 2 and 3: (Area isolation) Scenario 2 considers the isolation of the geographical area that encompasses actuators $\{4,9\}$ (see Fig.1). This implies that the actuators are turned off, i.e. the valves are closed. On the contrary, Scenario 3 assumes that the closest alternative actuator, that is $\{12\}$, can not be used either, removing it in the set of candidate actuators $\mathscr{A}_{C}$.

- Scenario 4: (Maintenance routine) This scenario simulates the development of maintenance works during the night in different areas of the network. Note that the considered time horizon $N$ is shorter than in the other scenarios (see Table I).

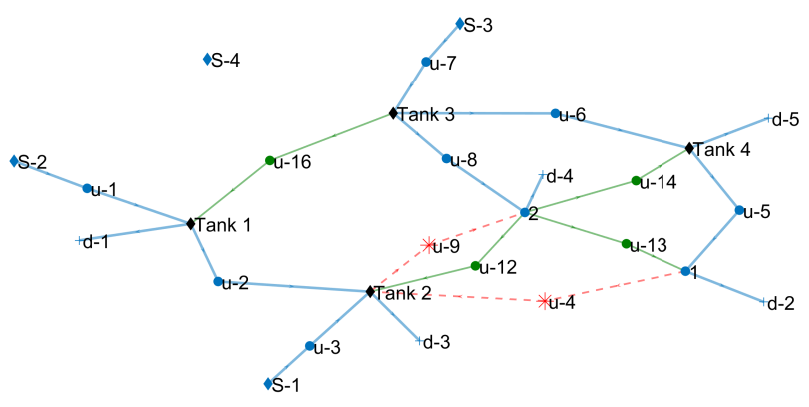

Fig. 2. Proposed system reconfiguration for Scenario 2 


\section{RESULTS}

The main results obtained for the different scenarios are summarized in Table II. Regarding the simulations, the mixed integer programs were solved using CPLEX optimizer and all the weighting matrices were set to the identity. Besides, a random initial position error was introduced at the initial time $k_{0}$ of each incidence.

TABLE II

RESULTS

\begin{tabular}{ccccc}
\hline Scenario & $\alpha_{N}\left(\mathscr{S}_{n}, x_{k_{0}}\right)$ & $\sigma_{N}\left(x_{k_{0}}\right)$ & $\alpha_{N}\left(\mathscr{S}_{e}, x_{k_{0}}\right)$ & $\mathscr{A}_{\boldsymbol{A}}$ \\
\hline 1 & 0.558 & 0.837 & 1134.5 & $\{13\}$ \\
2 & 0.626 & 0.939 & 1.139 & $\{12,13,14,16\}$ \\
3 & 0.626 & 0.939 & 1.139 & $\{11,13,14,15,16\}$ \\
4 & 5.089 & 7.634 & infeasible & $\{13,15,16\}$ \\
\hline
\end{tabular}

According to Table II all the considered scenarios yield a non-admissible expected performance $\alpha_{N}\left(\mathscr{S}_{e}, x_{k_{0}}\right)$ after the occurrence of the event, motivating thus the study of possible alternatives.

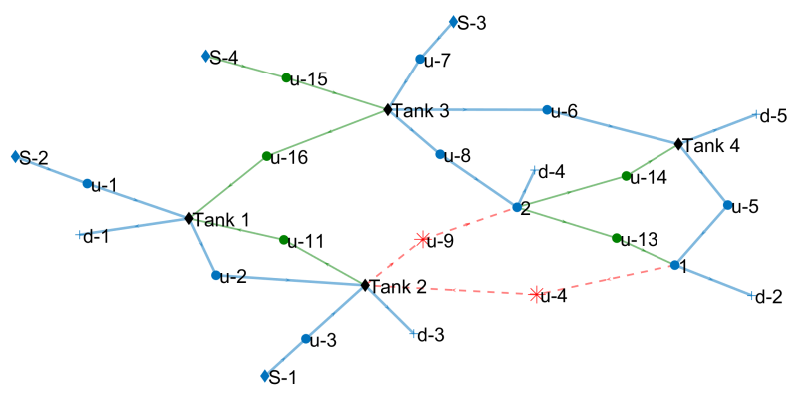

Fig. 3. Proposed system reconfiguration for Scenario 3

Figures 2 - 4 graphically represent the obtained system configurations for scenarios 2, 3 and 4, respectively. In each of these figures, the elements that have been shutted down are plotted in red, while the selected set of alternative actuators obtained in each case are depicted in green.

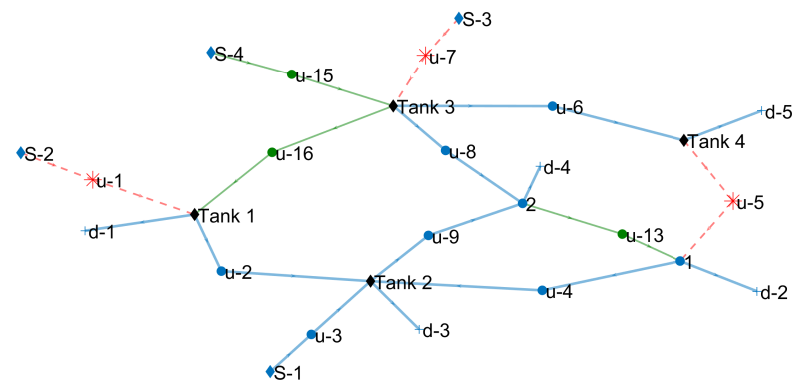

Fig. 4. Proposed system reconfiguration for Scenario 4

It must be remarked that although an economical criterion was employed in order to generate the reference trajectory, this was not taken into consideration in the actuators selection stage. This can lead to reconfiguration schemes with a high economical cost during the expected time horizon.

\section{CONCLUSIONS}

This paper formulates the system reconfiguration problem with back-up actuators, to which, despite some isolated cases, little attention has been paid from the automatic control community. The system reconfiguration only makes sense in scenarios where the nominal system performance is non-admissible. In this regard, the metrics used in order to evaluate the system performance are based on predictions over a time horizon. This work only considers the system reconfiguration problem and not the closed-loop control of the plant. It must be remarked that the combinatorial nature of the problem motivates to take advantage of the knowledge of the system in order to reduce the worst case execution time. The consideration of uncertainties in order to improve the robustness of the method, as well as developing a criterion in order to assess the weights associated to the different back-up actuators, were identified as future research directions.

\section{REFERENCES}

[1] M. Blanke, M. Kinnaert, J. Lunze, M. Staroswiecki, and J. Schröder, Diagnosis and fault-tolerant control, 3rd ed. Springer, 2016.

[2] J. Lunze and J. H. Richter, "Reconfigurable fault-tolerant control: A tutorial introduction," European journal of control, vol. 14, no. 5, pp. 359-386, 2008.

[3] S. Mishra, D. Das, and S. Paul, "A comprehensive review on power distribution network reconfiguration," Energy Systems, vol. 8, no. 2, pp. 227-284, 2017.

[4] S. Kalambe and G. Agnihotri, "Loss minimization techniques used in distribution network: bibliographical survey," renewable and sustainable energy reviews, vol. 29, pp. 184-200, 2014.

[5] D. Mignone, "Control and estimation of hybrid systems with mathematical optimization," Ph.D. dissertation, ETH Zurich, 2002.

[6] A. Casavola and E. Garone, "Fault-tolerant adaptive control allocation schemes for overactuated systems," International Journal of Robust and Nonlinear Control, vol. 20, no. 17, pp. 1958-1980, 2010.

[7] J. Tjonnas and T. A. Johansen, "Optimizing adaptive control allocation with actuator dynamics," in 2007 46th IEEE Conference on Decision and Control. IEEE, 2007, pp. 3780-3785.

[8] J. Stoustrup, "Plug \& play control: Control technology towards new challenges," European Journal of Control, vol. 15, no. 3-4, pp. 311330, 2009.

[9] H. A. Mahmoud, Z. Kapelan, and D. Savić, "Real-time operational response methodology for reducing failure impacts in water distribution systems," Journal of Water Resources Planning and Management, vol. 144, no. 7, pp. $04018029(1-14), 2018$.

[10] J. Grosso, C. Ocampo-Martínez, V. Puig, and B. Joseph, "Chanceconstrained model predictive control for drinking water networks," Journal of process control, vol. 24, no. 5, pp. 504-516, 2014.

[11] A. Bemporad and M. Morari, "Control of systems integrating logic, dynamics, and constraints," Automatica, vol. 35, no. 3, pp. 407-427, 1999.

[12] E. C. Kerrigan and J. M. Maciejowski, "Designing model predictive controllers with prioritised constraints and objectives," in Proceedings. IEEE International Symposium on Computer Aided Control System Design. IEEE, 2002, pp. 33-38.

[13] C. Ocampo-Martinez, A. Ingimundarson, V. Puig, and J. Quevedo, "Objective prioritization using lexicographic minimizers for mpc of sewer networks," IEEE Transactions on Control Systems Technology, vol. 16, no. 1, pp. 113-121, 2007.

[14] E. C. Kerrigan, A. Bemporad, D. Mignone, M. Morari, and J. M. Maciejowski, "Multi-objective prioritisation and reconfiguration for the control of constrained hybrid systems," in Proceedings of the 2000 American Control Conference. ACC (IEEE Cat. No. O0CH36334), vol. 3. IEEE, 2000, pp. 1694-1698.

[15] Y. Wang, V. Puig, and G. Cembrano, "Non-linear economic model predictive control of water distribution networks," Journal of Process Control, vol. 56, pp. 23-34, 2017. 